\title{
Protein kinase Sck1 is involved in trehalase activation by glucose and nitrogen source in the fission yeast Schizosaccharomyces pombe
}

\author{
T. Soto, J. Fernandez, J. Cansado, J. Vicente-Soler and M. Gacto \\ Author for correspondence: M. Gacto. Tel: +34 68 307100. Fax: +34 68363963. \\ e-mail: maga@fcu.um.es
}

Department of Genetics and Microbiology, Facultad de Biología, University of Murcia, 30071 Murcia, Spain
Trehalase activity is markedly enhanced upon addition of glucose and a nitrogen source to cells of the fission yeast Schizosaccharomyces pombe. This increase corresponds to a post-translational activation of the enzyme, which is controlled by CAMP-dependent and CAMP-independent pathways. Recent work has shown that overexpression of SCK1 in Schiz. pombe is able to suppress mutations that result in reduced Pka1 (CAMP-dependent protein kinase $\mathrm{A}$ activity, suggesting that Sck1 (suppressor of loss of CAMP-dependent protein kinase) might be a functional analogue of Pka1 in the fission yeast. Here, an analysis of the possible role of Sck1 in the activation of trehalase triggered by glucose and a nitrogen source is reported in cells that were deficient in either Pka1, Sck1 or both protein kinases. The results showed that, except in repressed cells, Sck1 probably mediates a CAMP-independent activation of trehalase following the signal(s) triggered by glucose and the nitrogen source. The absence of functional Sck1 in derepressed cells renders trehalase insensitive to activation by glucose and the nitrogen source even in the presence of Pka1, indicating that the Sck1-dependent, CAMP-independent pathway is the main signalling pathway controlling trehalase activation under derepression conditions. It is proposed that, during the activation of trehalase induced by glucose or a nitrogen source, the CAMP-Pka1 activation pathway previously characterized is to some extent parallel to this newly described one which includes Sck1 as phosphorylating enzyme. Neither of these two pathways, however, plays a key role in the heat-induced increase in trehalase activity.

Keywords: Schizosaccharomyces pombe, trehalase, signal transduction, protein kinase, sck1 mutants

\section{INTRODUCTION}

In Saccharomyces cerevisiae the pathway integrated by Ras, adenylate cyclase and cAMP-dependent protein kinase (protein $\underline{\text { kinase }} \underline{\mathrm{A}}$; Pka1) regulates many cellular processes. This pathway is required for growth and cell cycle progression (Matsumuto et al., 1982) and also for transduction of the glucose signal into changes in the transcriptional expression pattern of various genes (Cherry et al., 1989). The activation of this phosphorylating system is mediated by an effectorinduced signal which is transmitted via a cAMP peak (Mbonyi et al., 1988; Munder \& Küntzel, 1989). The

Abbreviations: Grp, glucose-repressible protein; Pka1, protein kinase $\underline{\mathbf{A}}$;

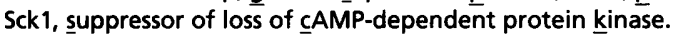

cAMP-Pka1 pathway also causes activation of cytoplasmic trehalase; this enzyme is phosphorylated by Pka1 and the active form then cleaves the disaccharide trehalose to form glucose (Uno et al., 1983; Thevelein, 1988).

In the fission yeast Schizosaccharomyces pombe, a similar, although Ras-independent, pathway plays a regulatory role in the control of catabolite repression of fructose 1,6-bisphosphatase (Fbp1; Hoffman \& Winston, 1991) and in trehalase activation after exposure to glucose and a nitrogen source (Carrillo et al., 1992; Soto et al., 1995a). There is recent evidence, however, that disruption of the gene encoding Pka1 does not block the activation of trehalase induced by either glucose or a nitrogen source in the fission yeast (Soto $e t$ 
al., 1995a, b), a result which supports the existence of a cAMP-independent signalling pathway alternative to that involving Pka1. Coincident with these observations, Jin et al. (1995) reported the presence in Schiz. pombe of the SCK1 gene, which is homologous to that encoding protein kinase Sch9 in Sacch. cerevisiae. At high copy number, SCK1 is able to suppress mutations in the cAMP-Pka1 pathway, which regulates FBP1 transcription, suggesting that Sck1 (suppressor of loss of cAMPdependent protein kinase) might form part of a control mechanism partially duplicating the Pka1 pathway in the fission yeast.

We have analysed the possibility that trehalase could be a common target for Sck1 and Pka1 by using disruptant strains of Schiz. pombe that lack either Sck1, Pka1 or both protein kinases. In this report, we show that Sck1 is indeed involved in activation of trehalase and that, except in repressed cells, its function appears to be required in the mechanism responsible for trehalase stimulation triggered by glucose and the nitrogen source.

\section{METHODS}

Yeast strains and media. Yeast strains used in this study are listed in Table 1 . The strains were cultured at $28^{\circ} \mathrm{C}$ with shaking in liquid medium containing either $2 \%(\mathrm{w} / \mathrm{v})$ glucose or $2 \%(\mathrm{w} / \mathrm{v})$ glycerol as a carbon source plus $0.6 \%$ yeast extract. When necessary, cultures were supplemented with Lhistidine, L-leucine, uracil (50 $\mathrm{mg} \mathrm{l}^{-1}$ each) and adenine (100 $\left.\mathrm{mg} \mathrm{l}^{-1}\right)$. Repressed cells were collected from cultures in the mid-exponential phase of growth in glucose-containing medium. Derepressed cells were harvested from either midexponential-phase cultures growing in glycerol or stationaryphase cultures grown in glucose-containing medium after glucose exhaustion. In all cases, the cells were washed and resuspended in $10 \mathrm{mM} \mathrm{MES} / \mathrm{KOH}$ buffer, $\mathrm{pH} 6.0$, at a concentration of $50 \mathrm{mg}$ wet wt $\mathrm{ml}^{-1}$ (approximately $8 \times 10^{8}$ cells $\mathrm{ml}^{-1}$ ).

Trehalase activation and enzyme assays. The above suspensions were incubated in a shaking water bath and allowed to equilibrate at $28^{\circ} \mathrm{C}$. After addition of $100 \mathrm{mM}$ glucose (zero time), samples were removed at timed intervals, the cells washed with the suspension buffer and cell extracts prepared, as previously described (Carrillo et al., 1992). When the concentration of glucose decreased to values lower than
$30 \mathrm{mM}$ (usually within $90 \mathrm{~min}$ after the initial addition of sugar), the remaining cell suspension was divided into halves and one half was supplemented with $10 \mathrm{mM}$ asparagine as nitrogen source. Aliquots of each suspension were then taken at various times and processed as above. The activation of trehalase upon heat shock was carried out in repressed cells as described by De Virgilio et al. (1990). Trehalase assays and protein determinations were performed as reported previously (Carrillo et al., 1994). Trehalase activity was expressed as nmol $\min ^{-1}$ (mg protein) ${ }^{-1}$.

Reproducibility of results. All the experiments described were repeated at least three times with similar results. Representative results are shown.

\section{RESULTS}

\section{Sck1 is required for trehalase activation in derepressed cells}

Trehalase can be activated transiently in derepressed wild-type cells of Schiz. pombe by glucose and also (in the presence of an energy source) by nitrogen sources. In both cases, trehalase activation is preceded by a cAMP peak (Carrillo et al., 1994; Soto et al., 1995c). To examine the contribution of Pka1 and Sck1 to the activation process, suspensions of cells from cultures growing in glycerol-containing medium which were disrupted in either PKA1, SCK1 or both genes were first pulsed with $100 \mathrm{mM}$ glucose. After $90 \mathrm{~min}$, when the glucose-induced response of trehalase was declining and less than one-third of the initial concentration of glucose remained in the suspension buffer, $10 \mathrm{mM}$ asparagine was added to half of the cells and the level of trehalase activity was again determined at timed intervals. The effect of the selected disruptions on the glucose- and nitrogen-source-induced trehalase activation is shown in Fig. 1. As compared with their corresponding controls, SCK1-disrupted cells did not show any significant trehalase stimulation after addition of the abovementioned inducers, irrespective of the presence or absence (double disruptant) of functional Pka1 (Fig. $1 \mathrm{a}, \mathrm{b})$. This indicates that Sck1 is needed for trehalase activation by glucose and a nitrogen source in derepressed cells. Similar results were obtained with derepressed cells from glucose-grown stationary-phase

Table 1. Schiz. pombe strains used in this study

\begin{tabular}{|c|c|c|}
\hline Strain & Genotype & Reference/source \\
\hline JZ633 & $h^{90}$ ade6-M216 leu1 ura4-D18 $\Delta p k a 1:: U R A 4^{+}$ & Maeda et al. (1994) \\
\hline JZ636 & $h^{+}$ade6-M210 leu1 ura4-D18 $\Delta p k a 1:: U R A 4^{+}$ & Maeda et al. (1994) \\
\hline $\mathrm{CHP} 429$ & $b^{-}$ade6-M216 his7-366 leu1-32 ura4-D18 & C. S. Hoffman* \\
\hline $\mathrm{CHP} 433$ & $\begin{array}{l}b^{-} \text {ade6-M216 his7-366 leu1-32 ura4-D18 } \Delta s c k 1- \\
1:: \text { HIS7 }^{+}\end{array}$ & C. S. Hoffman* \\
\hline $\mathrm{CHP} 453$ & $b^{-}$his7-366 leu1-32 ura4-D18 $\Delta p k a 1:: U R A 4^{+}$ & Jin et al. (1995) \\
\hline CHP458 & $\begin{array}{l}h^{-} \text {bis7-366 leu1-32 ura4-D18 } \Delta p k a 1:: U R A 4^{+} \\
\Delta s c k 1-1:: \text { HIS7 }^{+}\end{array}$ & Jin et al. (1995) \\
\hline
\end{tabular}

*Boston College, MA, USA. 


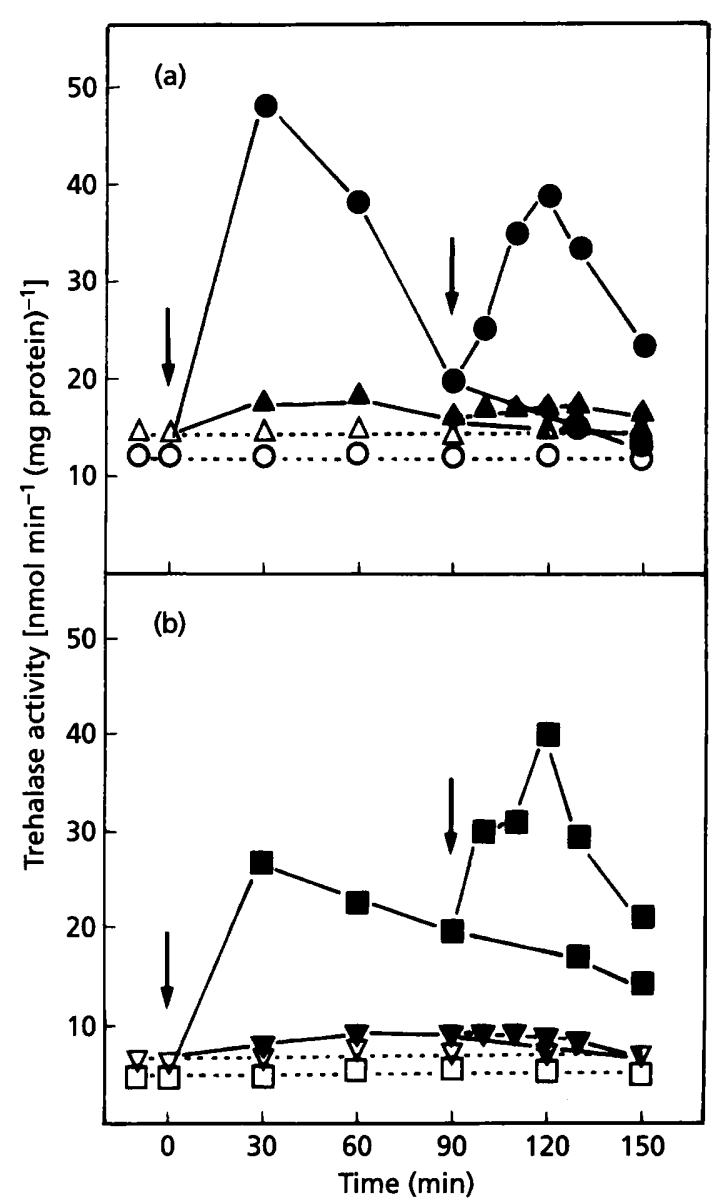

Fig. 1. Activation of trehalase in derepressed cells of Schiz. pombe from cultures growing in glycerol after addition of $100 \mathrm{mM}$ glucose (first arrow), and $10 \mathrm{mM}$ asparagine to half of cells (second arrow). (a) O. Strain CHP429 (PKA1 SCK1); $A$, strain CHP433 (PKA1 sck1). (b) 1 , Strain CHP453 (pka1 SCK1); $\nabla$, strain CHP458 (pka1 sck1). The concentration of glucose remaining in the suspension buffer at the time of addition of asparagine varied from 25 to $31 \mathrm{mM}$. Open symbols and dashed lines represent trehalase activity in corresponding control cells from cultures non-supplemented with glucose or a nitrogen source.

cultures after exhaustion of the sugar from the culture medium (Fig. 2).

\section{Pka1 function may account for nitrogen-source- induced trehalase activation in repressed cells}

We next examined the activation of trehalase induced by glucose and a nitrogen source in control, PKA1 and/or SCK1 disruptants under conditions of glucose repression. The results are shown in Fig. 3. In accordance with previous results suggesting that a glucose-repressible element is required in the glucose-induced signalling pathway, but not in that induced by a nitrogen source (Carrillo et al., 1992; Soto et al., 1995a, c), wild-type cells of Schiz. pombe lacked the glucose-induced activation of trehalase but still retained the ability to

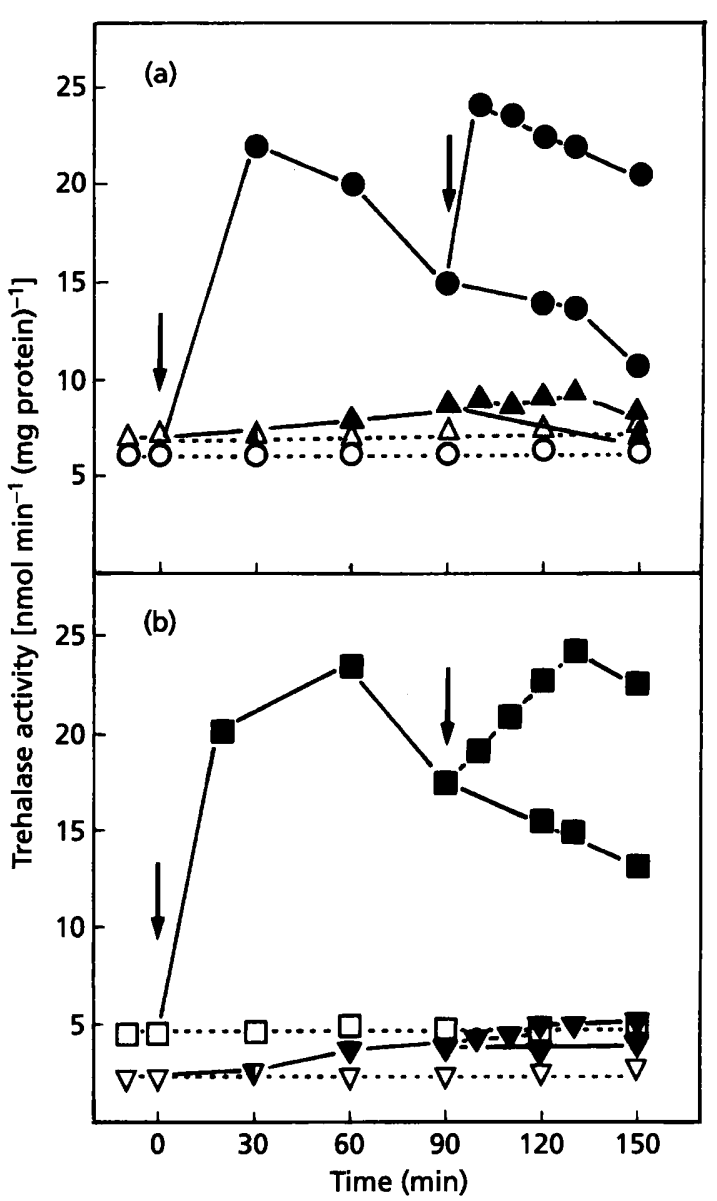

Fig. 2. Activation of trehalase in derepressed cells of Schiz. pombe from glucose-depleted stationary-phase cultures after addition of $100 \mathrm{mM}$ glucose (first arrow), and $10 \mathrm{mM}$ asparagine to half of cells (second arrow). (a) O. Strain CHP429 (PKA1 SCK1); $A$, strain CHP433 (PKA1 sck1). (b) $\square$, Strain CHP453 (pka1 SCK1); $\nabla$, strain CHP458 (pka1 sck1). The concentration of glucose remaining in the suspension buffer at the time of addition of asparagine varied from 22 to $30 \mathrm{mM}$. Open symbols and dashed lines represent trehalase activity in corresponding control cells from cultures non-supplemented with glucose or a nitrogen source.

increase trehalase activity in response to the addition of asparagine (Fig. 3a). Cells with SCK1 deleted gave results similar to those obtained in wild-type cells (Fig. 3a) whereas cells with both PKA1 and SCK1 deleted were unable to increase trehalase activity significantly after addition of either glucose or a nitrogen source (Fig. $3 b)$. This suggests that Pka1 might be responsible for the nitrogen-source-induced activation of trehalase in glucose-repressed cells. On the other hand, cells disrupted in PKA1 showed both glucose- and nitrogensource-induced activation of trehalase (Fig. $3 \mathrm{~b}$ ), a result which is in agreement with the notion that PKA1disrupted cells are constitutively derepressed even in the presence of glucose (Hoffman \& Winston, 1990, 1991; Byrne \& Hoffman, 1993). The activation of trehalase induced by glucose in pka1 SCK1 cells can be also interpreted as evidence for the presence of a glucose- 


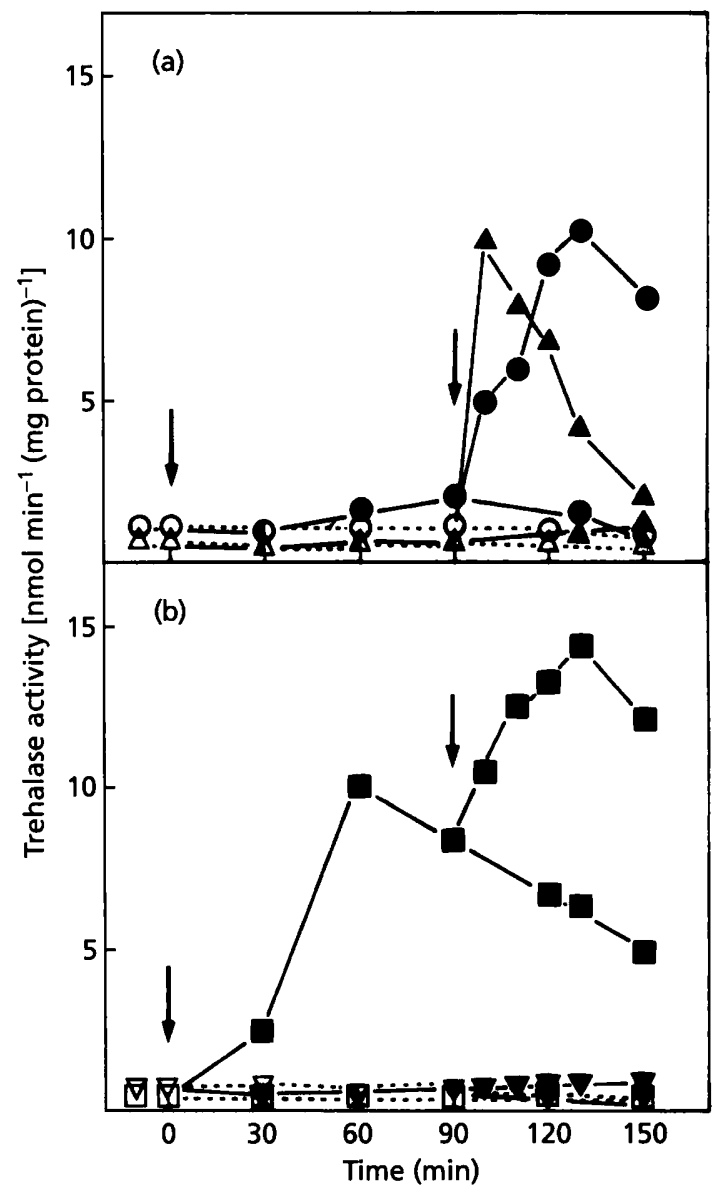

Fig. 3. Activation of trehalase in repressed cells of Schiz. pombe from cultures growing in glucose after addition of $100 \mathrm{mM}$ glucose (first arrow), and $10 \mathrm{mM}$ asparagine to half of cells (second arrow). (a) 0 , Strain CHP429 (PKA1 SCK1); $A$, strain CHP433 (PKA1 sck1). (b) ם, Strain CHP453 (pka1 SCK1); strain CHP458 (pka1 sck1). The concentration of glucose remaining in the suspension buffer at the time of addition of asparagine varied from 16 to $21 \mathrm{mM}$. Open symbols and dashed lines represent trehalase activity in corresponding control cells from cultures non-supplemented with glucose or a nitrogen source.

repressible protein $(\mathrm{Grp})$ in the signalling pathway that is missing in repressed cells.

\section{Pka1 and Sck1 are not members of the same signalling pathway}

The inability of $\mathrm{Pka} 1$ to activate trehalase in derepressed cells in the absence of Sck1 (Figs 1,2) might suggest that both kinases form part of a single pathway (with Sck1 downstream of $\mathrm{Pka} 1$ ) instead of merely sharing trehalase as a common substrate. However, the fact that $\mathrm{Pka} 1 \mathrm{can}$ be active in repressed cells in the absence of Sck1 is incompatible with the existence of such a single pathway. To further clarify this matter, we investigated the effect of cAMP on trehalase activation of disruptant cells. Because the addition of cAMP to the fission yeast has been shown to stimulate trehalase through ac-

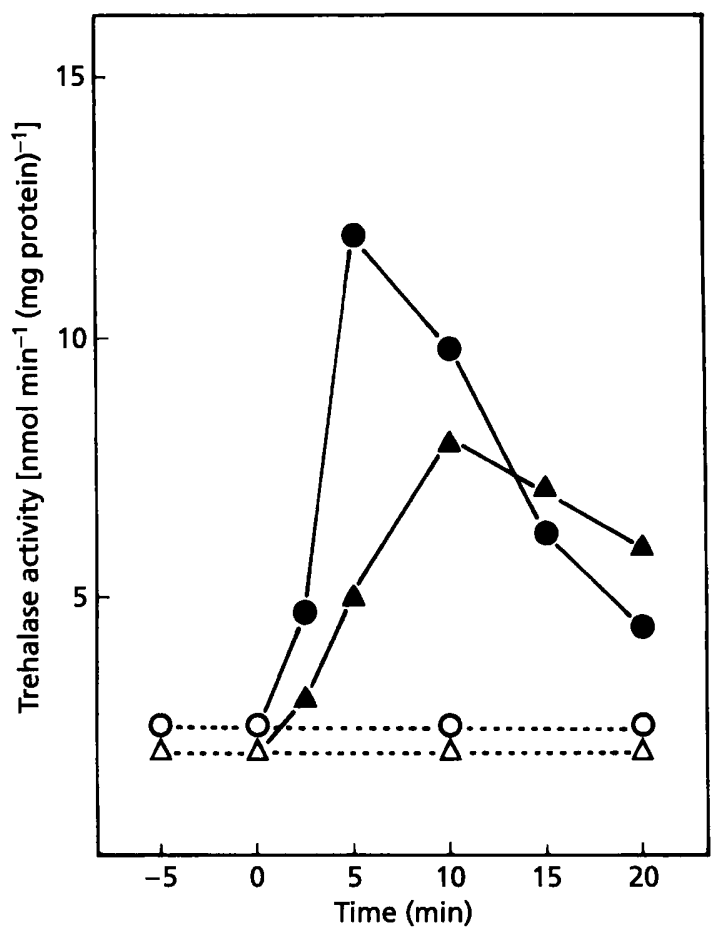

Fig. 4. Effect of the addition of CAMP on trehalase activity in repressed cells from strains CHP429 (PKA1 SCK1, O) and CHP433 (PKA1 sck1, $\Delta$ ). Cells suspended in incubation buffer were supplemented with $5 \mathrm{mM}$ CAMP at zero time and trehalase was determined at the indicated time intervals. Open symbols and dashed lines represent trehalase activity in corresponding control cells from suspensions nonsupplemented with CAMP.

tivation of Pka1 (Carrillo et al., 1992), we pulsed PKA1 sck1 cells with $5 \mathrm{mM}$ cAMP in an attempt to show the position of Sck1 relative to Pka1 in a supposed signalling pathway. As Fig. 4 indicates, trehalase activity increased after addition of exogenous cAMP in both control and SCK1-disrupted cells. This result, together with that showing that $\mathrm{Pka} 1$ can activate trehalase in repressed PKA1 sck1 cells (Fig. 3a), strongly implies that Pka1 activity is unlinked to Sck1 activity and, therefore, that the latter, rather than acting upstream or dowstream from Pka1 in the same signalling pathway, may activate trehalase independently.

\section{Neither Pka1 nor Sck1 are involved in the increase in trehalase activity induced by heat shock}

Previous studies indicate that trehalase activity increases markedly in Schiz. pombe on heat treatment. Although initial results obtained by De Virgilio et al. (1990) proposed that this increase was due to a posttranslational modification of the trehalase enzyme similar to that occurring after addition of glucose (i.e. phosphorylation), the heat-shock-induced response probably includes enhanced transcription of trehalase (Fernandez et al., 1997). We investigated whether a lack of Pka1 or Sck1 function could influence the trehalose 


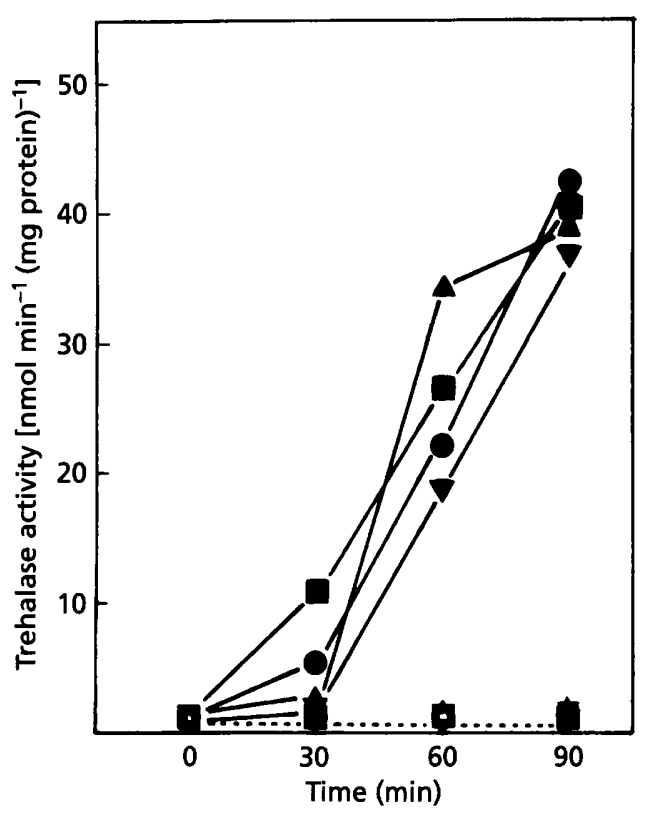

Fig. 5. Activation of trehalase by heat shock in strains CHP429 (PKA1 SCK1, O), CHP433 (PKA1 sck1, A), CHP453 (pka1 SCK1, $\square$ ) and CHP458 (pka1 sck1, $\nabla$ ). Cultures were grown at $28^{\circ} \mathrm{C}$ in glucose-containing medium to mid-exponential phase and then shifted to $40^{\circ} \mathrm{C}$ (zero time). Open symbols and dashed lines represent trehalase activity in corresponding control cultures maintained at $28^{\circ} \mathrm{C}$.

response to a heat treatment. As shown in Fig. 5, the increase in trehalase activity after a temperature shift from $28^{\circ} \mathrm{C}$ to $40^{\circ} \mathrm{C}$ was comparable in strains lacking PKA1, SCK1 or both genes. Hence, the corresponding gene products are of no critical importance for the heatshock-induced enhancement of trehalase activity. Similar results were obtained when derepressed cells of the above disruptant strains growing in glycerol were used (not shown).

\section{DISCUSSION}

This study shows that Sck1 most likely corresponds to the cAMP-independent protein kinase capable of activating trehalase, the existence of which was suspected in Schiz. pombe from earlier studies (Soto et al., 1995a, b). The analysis of the activation of trehalase induced by glucose and nitrogen source in cells selectively disrupted for PKA1 or SCK1 reveals that the two protein kinases do not appear to act in a single linear pathway. If both kinases were members of a same pathway, Sck1 should act downstream from $\mathrm{Pka} 1$ in derepressed cells, since PKA1 sck1 cells do not activate trehalase in the presence of the inducers (Figs 1a, 2a). However, the opposite should occur in repressed cells, because Pka1 is able to activate trehalase in these cells in the absence of Sck1 function (Fig. 3a). Moreover, the activation of trehalase after addition of cAMP to SCK1-disrupted cells is not congruent with the actuation of Sck1 downstream from

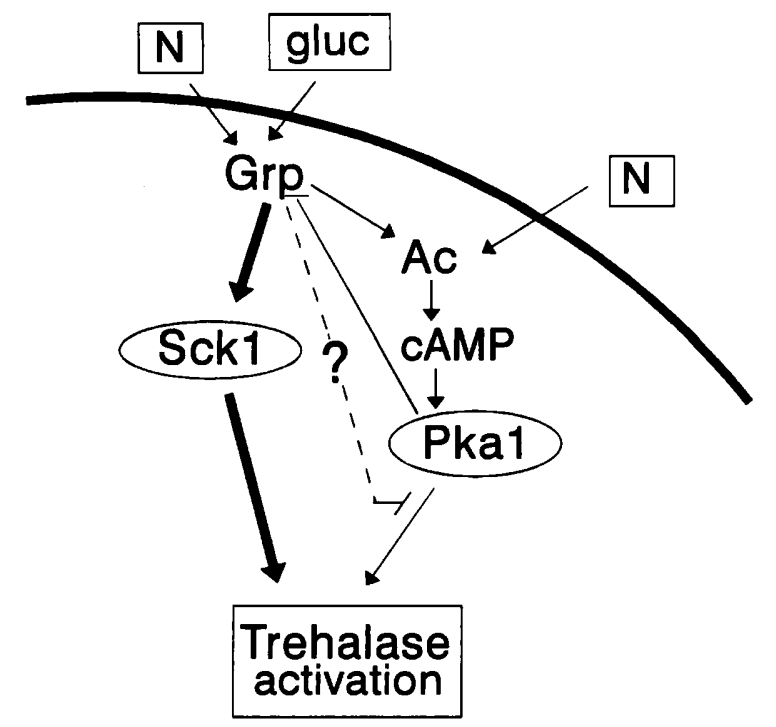

Fig. 6. Model for the action of the Pka1 and Sck1 pathways in trehalase activation in Schiz. pombe. gluc, glucose; N, nitrogen source; Grp, putative glucose-repressible protein; $A C$, adenylate cyclase. The scheme does not exclude the involvement of additional elements in the transmission of the input signals. See text for details.

Pka1. Therefore, we conclude that Pka1 and Sck1 are included in two different, largely independent trehalaseactivating pathways that converge on one (or more) common substrate(s). Furthermore, our results suggest that $\mathrm{Pka} 1$ is required for trehalase activation during repression whereas Sck1 is needed for trehalase activation under derepression conditions.

To explain the above results, we propose a branched model for trehalase activation in the fission yeast which also integrates observations made previously. According to this tentative model (Fig. 6), the signal produced by glucose would enter into the pathways triggering trehalase activation through a Grp, which appears to be needed for glucose signal transduction in Schiz. pombe (Carrillo et al., 1992). In contrast, the signal derived from nitrogen sources would show two possible points for interaction, one of them entering into the $\mathrm{Pka} 1$ pathway downstream from Grp in repressed cells (Soto et al., 1995a). The existence of the repressible Grp could explain the failure of glucose to activate trehalase in repressed cells and the controlled channelling of both glucose- and nitrogen-source-induced signals into one of the two alternative pathways in derepressed cells. This scheme provides an explanation for the fact that the Sck1 pathway is not functional for trehalase activation in repressed cells whereas it is operative in derepressed cells after addition of either glucose or nitrogen source. The model also explains how, since Pka1 negatively regulates the expression of genes controlled by catabolite-repression (Hoffman \& Winston, 1990, 1991; Byrne \& Hoffman, 1993), PKA1-disrupted cells behave as constitutively derepressed, and hence show trehalase 
activation by glucose even when they are actively growing on this sugar as carbon source (Fig. 3b).

In Sacch. cerevisiae, several studies with glucose repression and derepression mutants provide extensive evidence for the presence of such a putative Grp in the activation of the cAMP pathway by glucose (Thevelein, 1992). In Schiz. pombe, the mutually excluding action of the Sck1 and the Pka1 pathways on trehalase could simply rely on the impossibility of the simultaneous presence of both Grp (derepression) and Pka1 function (repression). Consistent with this is the demonstration that glucose inhibits the expression of some proteins through a cAMP signalling mechanism (Watanabe \& Yamamoto, 1996; Stettler et al., 1996). However, our observations also imply that the two pathways must somehow interact at more than just a final point, i.e before acting on trehalose as substrate. Support for this comes from results indicating that, in spite of the fact that the Pka1 pathway does not seem to play any significant role in trehalase phosphorylation in derepressed cells, the addition of glucose or a nitrogen source induces a transient peak in the cAMP level (Soto et al., 1995c). Consequently, some additional mechanism has to be present during derepression to control the dual regulation of trehalase and to prevent its activation by the Pka1 pathway in response to such additions. The nature of this control is unknown although a possible candidate is Grp, which might be not only repressed in the presence of Pka1 function but also under catabolite inactivation, with cAMP as a mediator in this effect (Holzer, 1976). Alternative explanations, such as a simple competitive inhibition between the two signalling pathways based on a differential affinity for trehalase, do not easily account for our results because they are unable to explain the lack of activation of trehalase in PKA1 sck1 derepressed cells. In any case, an important corollary of our present results is that a cAMP peak may be coincidental with, but not the cause of, trehalase activation in the fission yeast.

In earlier studies we suggested that Pka1 might be the only kinase able to activate trehalase in non-growing cells because the enzyme was apparently not activatable by glucose in the PKA1-disrupted strains JZ636 and JZ633 at stationary phase (Soto et al., 1995a, b). However, such an interpretation obviously has to be reconsidered in the light of the present results. A reexamination of the experiments that were performed with those two strains (which are auxotrophic for adenine) revealed that the culture media used for growth were not sufficiently supplemented to overcome the auxotrophy. When adenine $\left(100 \mathrm{mg} \mathrm{l}^{-1}\right)$ is supplied to the culture media before reaching stationary phase trehalase is activatable by glucose in both JZ636 and JZ633 strains, confirming that the apparent failure of trehalase activation in stationary phase was due to a decreased ATP pool for trehalase phosphorylation after exhaustion of the adenine present in the yeast extract, rather than to lack of Sck1 function. Therefore, the results are identical to those obtained in this work with strain CHP453 and serve to indicate that, contrary to our previous suggestion, Sck1, and not Pka1, phosphorylates, and thereby activates, trehalase in nongrowing cells provided ATP is available.

Cells harbouring a disruption in both SCK1 and PKA1 showed trehalase insensitive to activation by glucose and a nitrogen source, a situation that would be expected after effective removal of the pathways for trehalase activation. This outcome gives experimental support to the interpretation by Jin et al. (1995) that the extended lag in the growth of pka1 sck1 cells might be due to a block in the mobilization of trehalose that would deprive the cell of access to this energy reserve and delay exit from the stationary phase.

Our data additionally demonstrate that, unlike the phosphorylation underlying the activation induced by carbon and a nitrogen source, the increase in trehalase activity caused by heat shock does not require either Pka1 or Sck1 (Fig. 5). This reveals that, under stress conditions, additional pathways are involved in the regulation of trehalase and, hence, that other protein kinases may have a role in the control of trehalose breakdown in the fission yeast.

\section{ACKNOWLEDGEMENTS}

We thank C. S. Hoffman (Boston College, MA, USA) and M. Yamamoto (University of Tokyo, Japan) for kind provision of Schiz. pombe yeast strains. We are also grateful to Professor M. J. Walker for critical reading of the manuscript and to F. Garro for technical assistance. T.S. and J.F. are recipients of a fellowship from CajaMurcia, Spain. This work was supported in part by grant PB941151 from DGICYT, Spain.

\section{REFERENCES}

Byrne, S. M. \& Hoffman, C. S. (1993). Six git genes encode a glucose-induced adenylate cyclase activation pathway in the fission yeast Schizosaccharomyces pombe. J Cell Sci 105, 1095-1100.

Carrillo, D., Vicente-Soler, J. \& Gacto, M. (1992). Activation of neutral trehalase by fermentable sugars and cAMP in the fission yeast Schizosaccharomyces pombe. FEMS Microbiol Lett 98, 61-66.

Carrillo, D., Vicente-Soler, J. \& Gacto, M. (1994). Cyclic AMP signalling pathway and trehalase activation in the fission yeast Schizosaccharomyces pombe. Microbiology 140, 1467-1472.

Cherry, J. R., Johnson, T. R., Dollard, C., Shuster, J. R. \& Denis, C. L. (1989). Cyclic AMP-dependent protein kinase phosphorylates and inactivates the yeast transcriptional activator ADR1. Cell 56, 409-419.

De Virgilio, C., Simmen, U., Hottiger, T., Boller, T. \& Wienkem, A. (1990). Heat shock induces enzymes of trehalose metabolism, trehalose accumulation, and thermotolerance in Schizosaccharomyces pombe, even in the presence of cycloheximide. FEBS Lett 273, 107-110.

Fernandez, J., Soto, T., Vicente-Soler, J., Cansado, J. \& Gacto, M. (1997). Heat shock response in Schizosaccharomyces pombe cells lacking cyclic AMP-dependent phosphorylation. Curr Genet 31, 112-118.

Hoffman, C. S. \& Winston, F. (1990). Isolation and charac- 
terization of mutants constitutive for expression of the $f b p 1$ gene of Schizosaccharomyces pombe. Genetics 124, 807-816.

Hoffman, C. S. \& Winston, F. (1991). Glucose repression of transcription of the Schizosaccharomyces pombe fbp 1 gene occurs by a cAMP-signalling pathway. Genes Dev 5, 561-571.

Holzer, H. (1976). Catabolite inactivation in yeast. Trends Biochem Sci 1, 178-181.

Jin, M., Fujita, M., Culley, B. M., Apolinario, E., Yamamoto, M., Maundrell, K. \& Hoffman, C. S. (1995). Sck1, a high copy number suppressor of defects in the cAMP-dependent protein kinase pathway in fission yeast, encodes a protein homologous to the Saccharomyces cerevisiae SCH9 kinase. Genetics 140, 457-467.

Maeda, T., Watanabe, Y., Kunitomo, H. \& Yamamoto, M. (1994). Cloning of the Pka1 gene encoding the catalytic subunit of the cAMP-dependent protein kinase in Schizosaccharomyces pombe. J Biol Chem 269, 9632-9637.

Matsumoto, K., Uno, I., Oshima, Y. \& Ishikawa, T. (1982). Isolation and characterization of yeast mutants deficient in adenylate cyclase and cAMP-dependent protein kinase. Proc Natl Acad Sci USA 79, 2355-2359.

Mbonyi, K., Beullens, M., Detremerie, K., Geerts, L. \& Thevelein, J. M. (1988). Requirement of one functional RAS gene and inability of an oncogenic RAS variant to mediate the glucoseinduced cyclic AMP signal in the yeast Saccharomyces cerevisiae. Mol Cell Biol 8, 3051-3057.

Munder, T. \& Kuntzel, H. (1989). Glucose-induced cAMP signalling in Saccharomyces cerevisiae is mediated by $\mathrm{CDC} 25$ protein. FEBS Lett 242, 341-345.

Soto, T., Fernandez, J., Vicente-Soler, J., Cansado, J. \& Gacto, M. (1995a). Activation of neutral trehalase by glucose and nitrogen sources in Schizosaccharomyces pombe strains deficient in cAMPdependent protein kinase activity. FEBS Lett 367, 263-266.

Soto, T., Fernandez, J., Cansado, J., Vicente-Soler, J. \& Gacto, M. (1995b). Glucose-induced, cyclic-AMP-independent signalling pathway for activation of neutral trehalase in the fission yeast Schizosaccharomyces pombe. Microbiology 141, 2665-2671.

Soto, T., Fernandez, J., Vicente-Soler, J., Cansado, J. \& Gacto, M. (1995c). Nitrogen-source-induced activation of neutral trehalase in Schizosaccharomyces pombe and Pachysolen tannophilus: role of cAMP as second messenger. FEMS Microbiol Lett 132, 229-232.

Stettler, S., Warbrick, E., Prochnik, S., Mackie, S. \& Fantes, P. (1996). The wis1 signal transduction pathway is required for expression of cAMP-repressed genes in fission yeast. J Cell Sci 109, 1927-1935.

Thevelein, J. M. (1988). Regulation of trehalase activity by phosphorylation-dephosphorylation during developmental transition in fungi. Exp Mycol 12, 1-12.

Thevelein, J. M. (1992). The RAS-adenylate cyclase pathway and cell cycle control in Sacharomyces cerevisiae. Antonie Leeuwenhoek 62, 109-130.

Uno, I., Matsumoto, K., Adachi, K. \& Ishikawa, T. (1983). Genetic and biochemical evidence that trehalase is a substrate of cAMPdependent protein kinase in yeast. J Biol Chem 258, 10867-10872.

Watanabe, Y. \& Yamamoto, M. (1996). Schizosaccharomyces pombe $p c r 1^{+}$encodes a CREB/ATF protein involved in regulation of gene expression for sexual development. Mol Cell Biol 16, $704-711$

Received 5 December 1996; revised 5 February 1997; accepted 19 February 1997. 